\title{
Disección aórtica asintomática en una paciente con síndrome de Turner, válvula aórtica bicúspide y recoartación aórtica
}

\author{
Héctor Alejo Orellano \\ Mirta Casella \\ Salvador Vicente Spina
}

Servicio de Ecocardiografía. Hospital Aeronáutico Central. Buenos Aires. Argentina

Recibido: 30/05/2020

Aceptado: 22/06/2020

En línea: 31/07/2020

Citar como: Orellano HA, Casella M, Spina SV. Disección aórtica asintomática en una paciente con síndrome de Turner, válvula aórtica bicúspide y recoartación aórtica. Rev Ecocar Pract (RETIC). 2020 (Jul); 3 (2): 33-35. doi: 10.37615/retic.v3n2a10.

Cite this as: Orellano HA, Casella M, Spina SV. Asymptomatic aortic dissection in a patient with Turner syndrome, bicuspid aortic valve, and aortic recoarctation. Rev Ecocar Pract (RETIC). 2020 (Jul); 3 (2): 33-35. doi: 10.37615/retic.v3n2a10.

\section{Palabras clave}

$\triangleright$ Disección aórtica

$\triangleright$ Síndrome de Turner

$\triangleright$ Coartación aórtica

$\triangleright$ Válvula aórtica bicúspide

Keywords

$\triangleright$ Aortic dissection

$\triangleright$ Turner syndrome

$\triangleright$ Aortic coarctation

$\triangleright$ Bicuspid aortic valve

\section{RESUMEN}

Se presenta el caso de una paciente de 47 años con síndrome de Turner, portadora de válvula aórtica bicúspide y antecedente de cirugía por coartación aórtica en la infancia, a quien se descubre durante un estudio de rutina una disección de la aorta ascendente asociada a insuficiencia aórtica severa secundaria a prolapso de dos de las valvas y a recoartación aórtica.

El síndrome de Turner se asocia con complicaciones cardiovasculares potencialmente graves. El seguimiento clínico y con técnicas de imagen es fundamental para intentar prevenirlas

ABSTRACT
We present the clinical case of a 47-year-old female patient with Turner syndrome, bicuspid aortic valve, and
previous history of surgery of aortic coarctation in the childhood. During a routine study, ascending aorta dis-
section associated with severe aortic insufficiency secondary to valve prolapse and aortic recoarctation were
found.
Turner syndrome is associated with potentially serious cardiovascular complications. Clinical and imaging follow-
up are essential to prevent them.

\section{Presentación del caso}

Una paciente de 47 de años acudió a los consultorios externos de Ecocardiografía para la realización de una ecocardiografía transtorácica (ETT) rutinaria de control. Como antecedentes relevantes presentaba síndrome de Turner (ST), había sido intervenida de coartación aórtica (CoAo) a los 7 años y es portadora de una válvula aórtica bicúspide (VAB) con estenosis leve.

La ecocardiografía evidenció un ventrículo izquierdo (VI) levemente dilatado con engrosamiento leve del septum interventricular y fracción de eyección conservada, así como dilatación importante de la raíz aórtica y la aorta ascendente (AA) proximal (Vídeo 1).

Con Doppler color se observa un flujo de insuficiencia aórtica (IAo) que impresionaba ser significativo. Focalizando la imagen a nivel de la AA, se observa una franca dilatación aneurismática de la misma, con un diámetro mayor de $7,5 \mathrm{~cm}$ (Figura 1). En ventana apical de tres cámaras se pudo observar con mayor detalle la $V A B$, que presentaba un prolapso marcado de dos de las valvas con jet de IAo significativo (Vídeo 2, Vídeo 3 y Figura 2).
En el eje supraesternal largo se observa un flap de disección aórtica (DAo) y la presencia de re-CoAo con gradiente máximo de hasta $77 \mathrm{mmHg}$ (Vídeo 4, Vídeo 5 y Figura 3).

La paciente fue derivada a un centro de alta complejidad donde se le realizó una angio-TC aórtica, que evidenció a nivel de la raíz aórtica un diámetro de $55 \times 46 \mathrm{~mm}$ y en AA un diámetro de $75 \times 69 \mathrm{~mm}$.

Además, confirmó el hallazgo de DAo que se extiende desde la raíz, pasando por AA, hasta el cayado aórtico, de una longitud aproximada de $98 \mathrm{~mm}$, siendo la luz verdadera de $48 \times 19 \mathrm{~mm}$, sin compromiso del tronco braquiocefálico, ni la subclavia izquierda.

Por último, se observa una CoAo con un anillo de $13 \mathrm{~mm}$ en relación con una aorta descendente de $20 \mathrm{~mm}$ (Figura 4 y Figura 5).

La paciente fue intervenida exitosamente, realizándose un procedimiento de recambio de raíz de aorta con cirugía de Bentall-Bono, con prótesis valvular mecánica $\mathrm{N}^{\circ} 23$ y a nivel de AA una prótesis recta de dacrón N. 30 , quedando pendiente para un segundo tiempo quirúrgico la resolución de la re-CoAo. 


\section{Estudio por imagen}

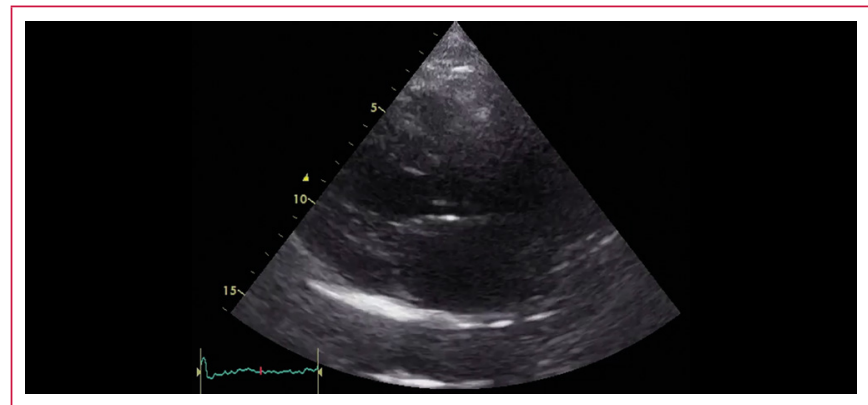

Vídeo 1. ETT, eje largo paraesternal. Se observa dilatación leve del VI con fracción de eyección conservada, hipertrofia leve del septum interventricular y dilatación de la raíz aórtica y aorta ascendente proximal

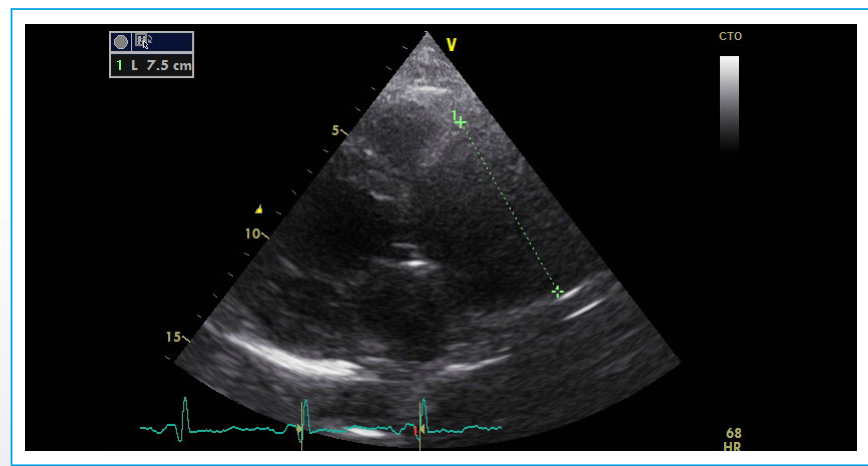

Figura 1. ETT, eje largo paraesternal izquierdo, focalizado en la raíz aórtica que evidencia dilatación severa del vaso

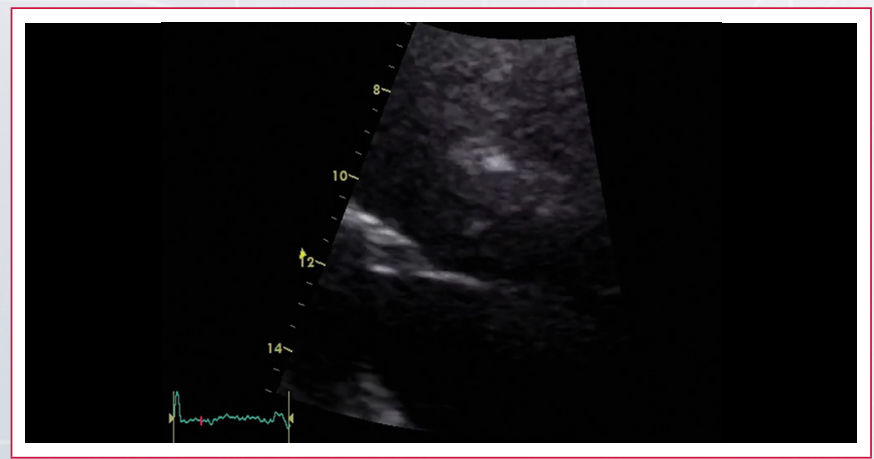

Vídeo 2. ETT, vista apical de tres cámaras con modo zoom en el tracto de salida del VI (TSVI). Se observa prolapso de dos velos de la válvula aórtica

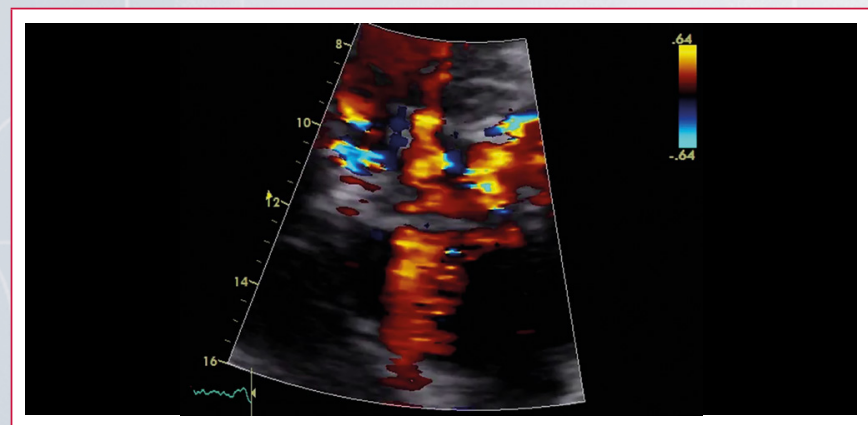

Vídeo 3. ETT, vista apical de tres cámaras con modo zoom y Doppler color en el TSVI. Se observa insuficiencia aórtica severa

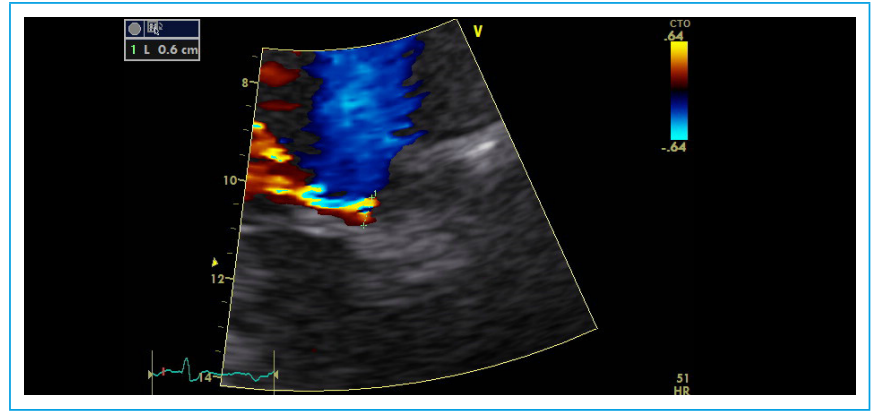

Figura 2. ETT a nivel del TSVI, con zoom y Doppler color a nivel valvular aórtico, en el que se realiza la medición de la vena contracta de la insuficiencia aórtica

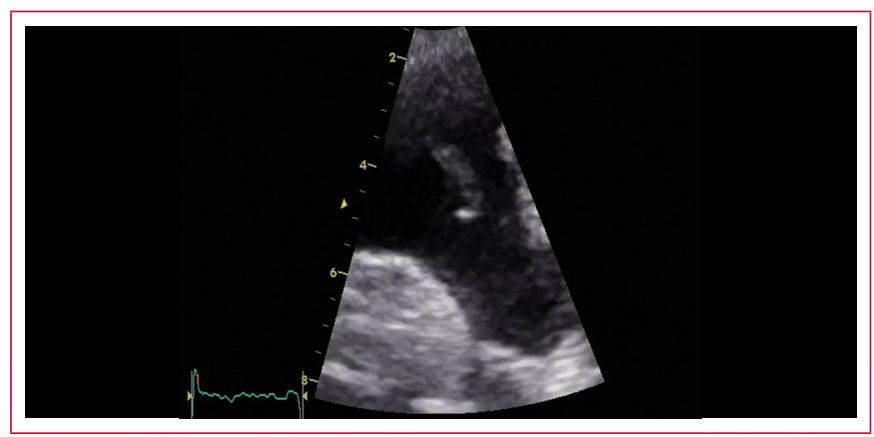

Vídeo 4. ETT, eje largo supraesternal. Se observa el flap de disección aórtica entre el cayado aórtico y la aorta torácica descendente

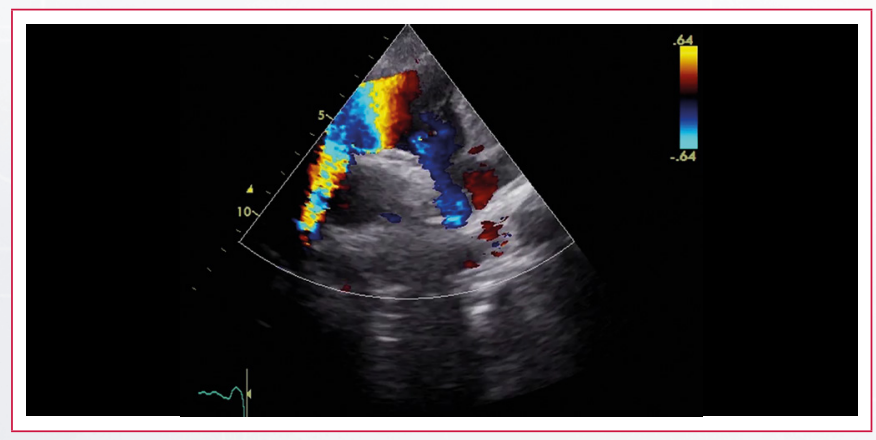

Vídeo 5. EET, eje largo supraesternal con Doppler color. Se aprecia un flujo marcadamente turbulento entre el cayado aórtico y la aorta torácica descendente que corresponde a disección aórtica y a recoartación de la aorta descendente

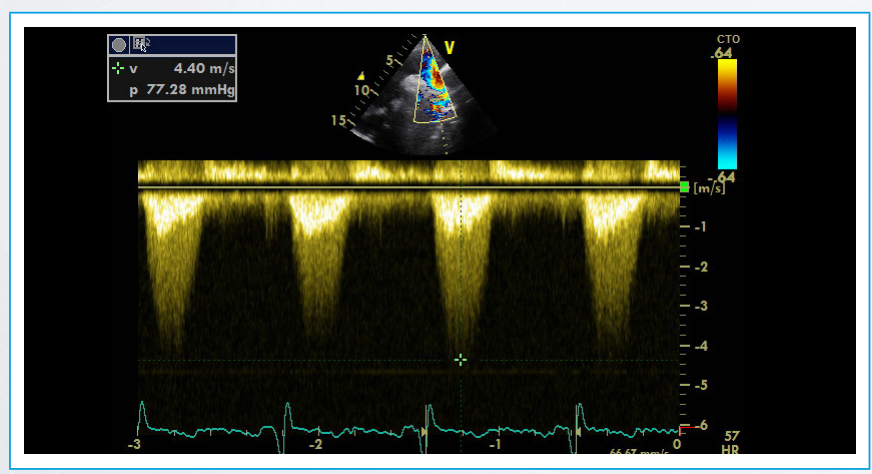

Figura 3. ETT, eje largo supraesternal, con la muestra de volumen del Doppler continuo en la aorta torácica descendente. Se obtienen velocidades y gradientes severamente aumentados, correspondientes a una recoartación aórtica 


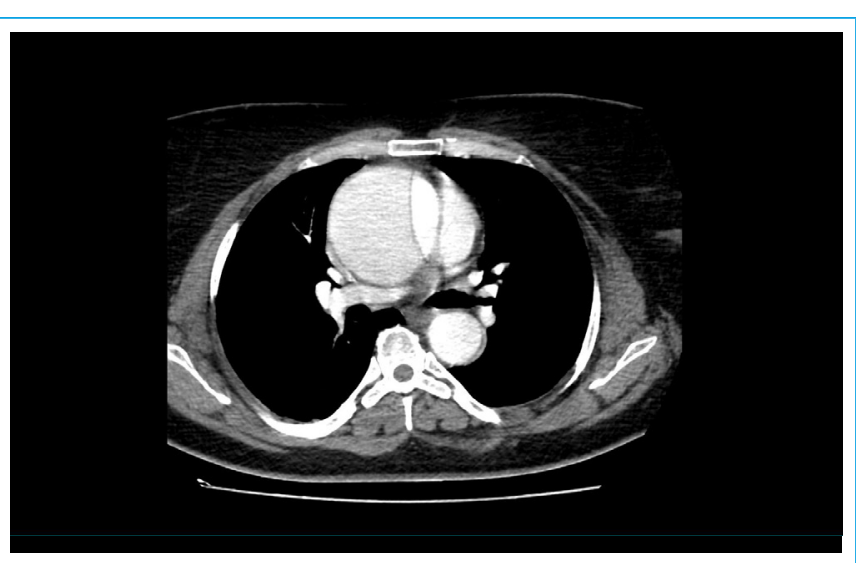

Figura 4. Angio-TC de aorta torácica. Corte transversal donde se observa la disección aórtica con la luz falsa (de mayor tamaño) y luz verdadera (de menor tamaño)

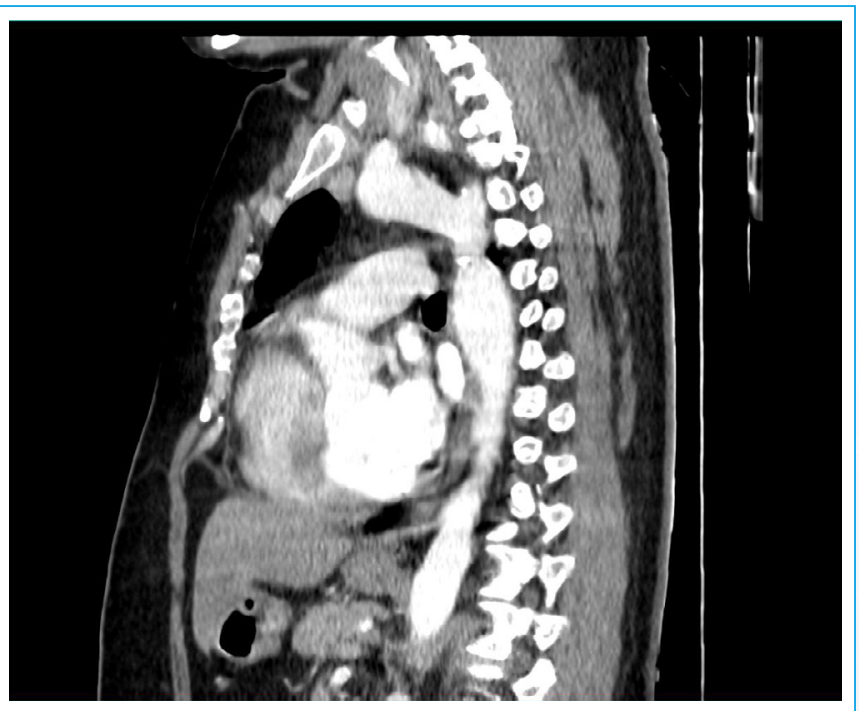

Figura 5. Angio-TC de aorta torácica. Corte sagital, con recoartación de la aorta torácica descendente

\section{Discusión}

El síndrome de Turner (ST) es un trastorno genético producido por la ausencia total o parcial del cromosoma X. Es la única monosomía compatible con la vida $y$ afecta a 1 de cada 2.000 niñas.

Para su diagnóstico se requiere la presencia de alteraciones fenotípicas típicas, que incluyen estatura baja, falla gonadal y defectos cardiovasculares congénitos, así como un cariotipo anormal. Las afecciones cardiovasculares son la principal causa de muerte prematura y comorbilidad en estas pacientes, pudiendo llegar a ser tres veces mayor que en la población general(1,2).

Las pacientes presentan un riesgo cardiovascular global aumentado, que en la actualidad sigue siendo motivo de discusión, pareciendo guardar relación con el déficit de estrógeno y la haploinsuficiencia del cromosoma $X^{(3-5)}$.

La válvula aórtica bicúspide (VAB) tiene una prevalencia del 30\% en esta población, mientras que la coartación aórtica (CoAo) está presente en el 12\% de estas pacientes.
La disección aórtica (DAo) es una complicación rara, que suele ser fatal en caso de no intervenirse. Tiene una incidencia estimada de 40 casos cada 10.000 pacientes ${ }^{(6)}$. Histopatológicamente se ha observado la presencia de necrosis quística de la capa media vascular, a su vez, se constató la presencia de alteración en elasticidad aórtica similares a lo observado en el síndrome de Marfan $^{(7,8)}$. La DAo suele ser silente y ocurre con diámetros aórticos inferiores a los observados en la población general. Los factores de riesgo para DAo son la coexistencia de VAB, CoAo, dilatación aórtica, hipertensión arterial y linfedema fetal; no obstante, entre el $10-25 \%$ de los casos sólo presentaban como factor predisponente el ST.

Finalmente, se debe recordar que es importante realizar la valoración de los diámetros aórticos indexados a la superficie corporal de forma sistemática, principalmente en este grupo de pacientes, dada su estatura baja.

\section{Conclusión}

En el síndrome de Turner las complicaciones cardiovasculares marcan el pronóstico del paciente. Para su seguimiento las técnicas de imagen cardiovascular juegan un papel fundamental. Los hallazgos histopatológicos y hemodinámicos sugieren un comportamiento muy similar a los observados en el síndrome de Marfan. No existe un consenso amplio de cómo debería realizarse el seguimiento de estos pacientes, por lo que todos los casos deben individualizarse e, idealmente, discutirse en un Heart Team.

\section{Ideas para recordar}

- El síndrome de Turner se asocia a afecciones cardiovasculares potencialmente graves.

- Tiene una prevalencia de válvula aórtica bicúspide del 30\% y de coartación aórtica en el $12 \%$ de los casos.

- El seguimiento debe ser clínico y con técnicas de imagen (ecocardiografía y tomografía).

- Es mandatorio indexar los diámetros aórticos a la superficie corporal.

\section{Bibliografía}

1. Bondy C. Congenital cardiovascular disease in Turner syndrome. Congenital Heart Disease 2008; 3 (1): 2-15

2. Bondy C. Aortic dissection in Turner syndrome. Current Opinion in Cardiology 2008; 23 (6): 519-526.

3. Donato B, Ferreira M. Cardiovascular risk in Turner syndrome. Revista Portuguesa de Cardiologia 2018; 37 (7): 607-621.

4. Kim H, Gottliebson W, Hor K, et al. Cardiovascular anomalies in Turner syndrome: Spectrum, prevalence, and cardiac MRI findings in a pediatric and young adult population. American Journal of Roentgenology 2011; 196 (2): 454-460.

5. Turtle $E$, Sule $A$, Bath $L$, et al. Assessing and addressing cardiovascular risk in adults with Turner syndrome. Clinical Endocrinology 2013; 78 (5): 639-645.

6. Kozlowska-Wojciechowska M, Jez W, Zdrojewski T, Chwojnicki K. Are young women with Turner syndrome at greater risk of coronary artery disease? European Journal of Cardiovascular Prevention \& Rehabilitation 2006; 13 (3): 467-469.

7. Trolle $C$, Hjerrild $B$, Cleemann $L$, et al. Sex hormone replacement in Turner syndrome. Endocrine 2011; 41 (2): 200-219.

8. Pees C, Heno J, Häusler G, et al. Aortic elasticity deterioration proves intrinsic abnormality of the ascending aorta in pediatric Turner syndrome unrelated to the aortic valve morphology. Heart and Vessels 2018; 33 (11): 1350-1357. 